GANIT J. Bangladesh Math. Soc. (ISSN 1606-3694) 35 (2015) 57-71

\title{
COMPRESSIBILITY EFFECTS IN 2D WALL HEATING MICROCHANNEL FLOW
}

\author{
Md. Tajul Islam \\ Department of Mathematics, Begum Rokeya University, Rangpur, Bangladesh \\ Email: tajul000@yahoo.com
}

Received 13.11.2014

Accepted 28.03.2015

\begin{abstract}
In this article we present a numerical solution of the Navier-Stokes equations and energy equation in parallel plate microchannels with the first order slip boundary conditions on the walls, adopting control volume scheme of CFD technique. Wall heating condition was considered on the walls. Noslip boundary conditions for compressible and incompressible flows were also solved to compare the effect of slip conditions. Compressibility effects were also investigated for compressible slip and compressible noslip flow conditions. A series of simulations were performed for different heights and lengths of channels and pressure ratios. Results are presented in graphs and tables and are compared with the available analytical and experimental results. It was found that the friction constants are the highest for noslip compressible flow and lowest for the slip flow against pressure ratio and mach numbers. Friction constant decreases continuously for compressible slip flow but it approaches to an asymptotic value of 96 for compressible noslip flow for the decrease of aspect ratio.
\end{abstract}

Keywords: Microchannel, Knudsen number, Slip flow, Temperature jump, Friction constant

\section{Introduction}

A systematic research effort in micromechanics devices started in the late 1980's. In response to the rapid progress in design and fabrication of microelectromechanical systems (MEMS) such as microducts, micronozzles, micropumps, microturbines and microvalves and so on, the need for understanding the momentum and heat transfer in microchannels is essential. Fluid flows in these devices differ from those in macroscopic devices. The operation of these devices cannot always be predicted from conventional flow models. This emerging field has generated strong interest and became a rapidly developing research field. This is because these channels have potential applications in diverge areas such as microelectronics, biological chips, microheat exchangers, etc. A large number of recent investigations study the fundamentals of microchannel flow and the development is great but not adequate.

The Knudsen number, a non-dimensional parameter, determines the degree of rarefaction and the degree of validity of the continuum model. It is defined as the ratio of the mean free path $\lambda=\frac{k_{B} T}{\sqrt{2} \pi p \sigma^{2}}$ over a characteristic length $\left(D_{h}=2 H\right)$ i.e. $K n=\frac{\lambda}{D_{h}}$. Thus the Knudsen number is 
expressed as $K n=\frac{k_{B} T}{\sqrt{2} \pi p \sigma^{2} D_{h}}$. In the limit of zero Knudsen number, the transport terms in the continuum momentum and energy equations are negligible, and the Navier-Stokes equations then reduce to the inviscid Euler equations. As $K n$ increases, rarefaction effects become more important, and continuum approach breaks down. A classification of the different flow regimes is given by George and Ali [5] as follows:

- for $K n \leq 0.01$ the fluid can be considered as a continuum

- for $K n \geq 0$ (10), it is considered as a free-molecular flow.

A rarefied gas can be considered neither as absolute continuous medium nor a free-molecular flow in the Knudsen number range between 0.01 and 10. In that region a further classification is needed, i.e.,

- $\quad$ slip flow $(0.01<K n<0.1)$, and

- transition flow $(0.1<K n<10)$

The different Knudsen number regimes are determined empirically and thus the limits between the different Knudsen number regimes may depend on the problem geometry.

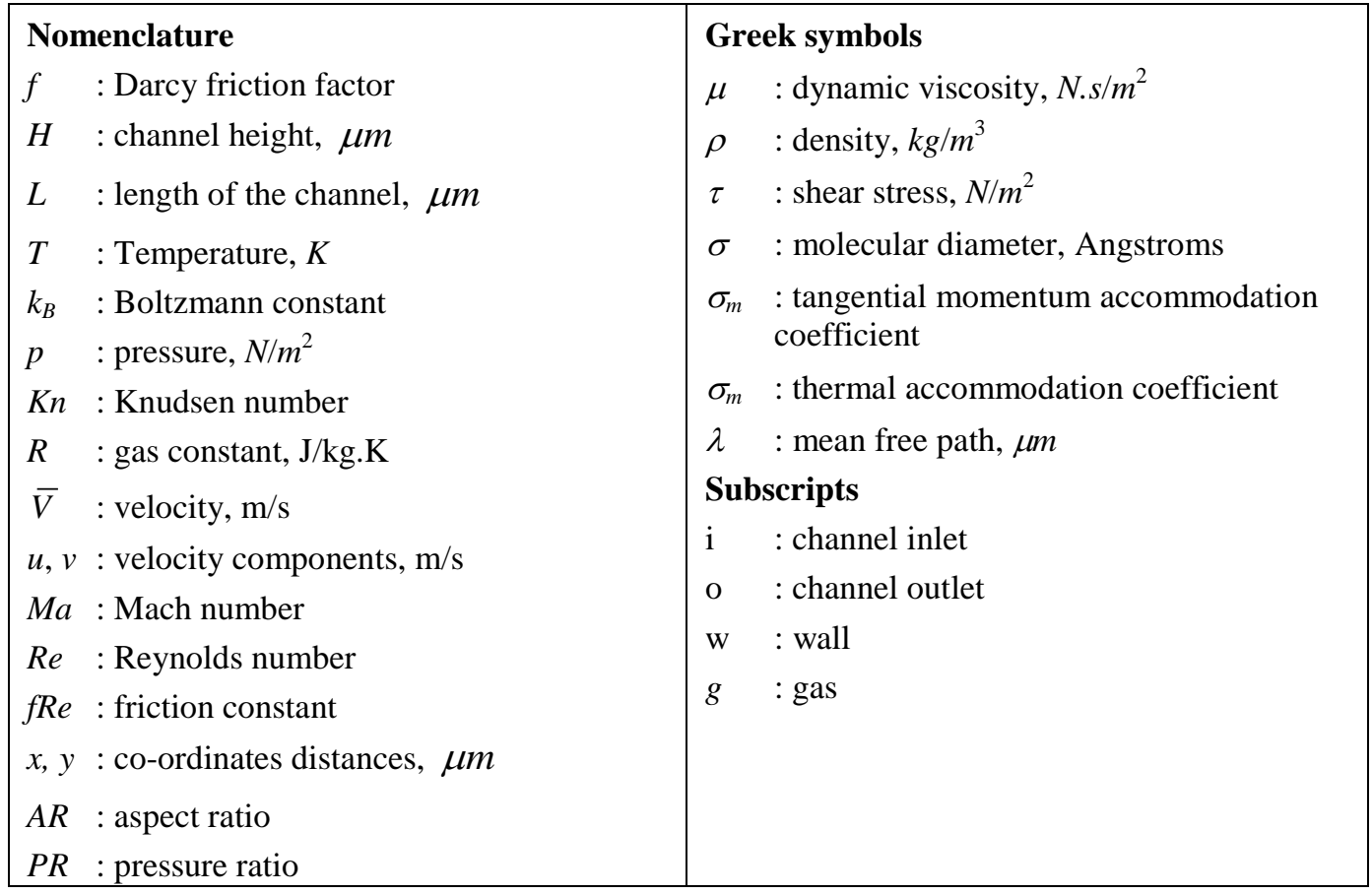

Giacomo Puccetti et al. [1] investigated 2D velocity profile in laminar regime of water flow through glass microchannels experimentally. The results have evidenced that $\mu \mathrm{PIV}$ is able to determine the velocity profile within the glass microchannel with an average relative difference 
with respect the theoretical values of the order of $1-4 \%$ depending on the aspect ratio of the channel in the central part of the channels.

Hamidi and Ouederni [2] studied three-dimensional flow behavior in straight rectangular microchannels with noslip boundary condition. The simulation results showed that geometric parameters have an effect on the velocity distribution in rectangular microchannels and it agreed well the he Poiseuille laminar flow theory.

Khaleel and Chunpei [3] studied asymptotic solutions of compressible gaseous flow through a three-dimensional straight uniform rectangular microchannel using higher order velocity slip boundary conditions. The analytical results of the first and second order velocity slip boundary conditions are compared with a benchmark case and there are appreciable differences between them.

Aniskin et al. [4] conducted experimental investigation to determine the friction factor in microchannels. Glass microchannels with a circular cross section, diameters of 34.5, 33.6, and 24.5 $\mu \mathrm{m}$, and different lengths are studied in the experiments. The results obtained are in good agreement with theoretical predictions for the case of a developed laminar flow in circular channels, but the use of the method of two channels has some constraints.

The pioneer investigators in the study of gaseous flow in microchannels are Maxwell [6], Smoluchowski [7], Arkilic et al. [8], Pong et al. [9], Ali and George [10], Schaaf and Chambre [11], Gad-el-Hak [12], Kakac [13], H. van den Berg et al. [14]. Most of the studies are considered incompressible because of simplicity and low Mach number flows. The studies of compressible flow, without the incorporation of rarefied behavior, have been conducted by Prud'homme et al. [15].

In spite of large number of investigations are performed so far, a lot of contradictory results are obtained and a large part of the behavior of the flow properties and the physical laws governing the fluid flow and heat transfer in microgeometries are uncovered. Two dimensional flow in parallel plate channel is the limiting case of rectangular ducts which are widely used in microfluidic devices. Though microchannel flows are low Mach number flows but these flows become compressible when their Mach number is less than 0.3. The objective of this study is to investigate the compressibility effect of compressible slip and compressible noslip flows and make a comparison among the obtained results. The solution is based on the concept of continuum approach on Navier-Stokes equations.

\section{Model Development}

\subsection{Problem Statement}

We considered nitrogen gas flow through parallel plate channel in Cartesian co-ordinates. Fig. 1 shows the geometry and co-ordinates of the microchannel. 
The flow domain is bounded by $0 \leq x \leq L$ and $-H / 2 \leq H / 2$, where $L$ and $H$ are the length and height of the channel. We defined aspect ratio as $A R=\frac{L}{H}$, for different lengths and heights where $750 \mu m \leq L \leq 6000 \mu m$ and $2.5 \mu m \leq H \leq 20 \mu m$. Pressure ratio was defined as $P R=\frac{p_{i}}{p_{0}}$, where pressure ratio ranges from 2 to 4 . All the dimensions are expressed in SI units.

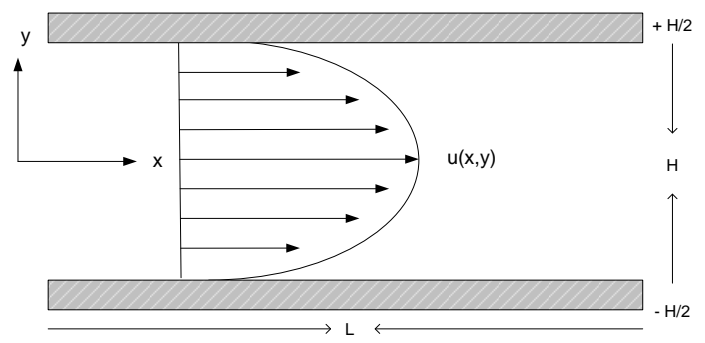

Fig. 1. Schematic diagram of the channel

We used two types of models; model 1 and model 2. In model 1, the channel height and length were fixed, $A R=300$, and pressure ratio ranges from 2 to 4 . The corresponding results are discussed and depicted by tables and figures from Fig. 2 to Fig. 6. Model 2 was designed by keeping height fixed, $P R=2$ and varying channel lengths from $750 \mu m$ to $6000 \mu m$ and the results are discussed and described by Figs. 7 - 10.

\subsection{Governing Equations}

The 2D gas flow and heat transfer is assumed to be steady and laminar. The Three basic laws of conservation of mass, momentum and energy are solved for both compressible and incompressible flows for Newtonian fluid. The compressible forms of the governing equations are expressed in following form and were simulated with the help of state equation $p=\rho R T$.

Continuity:

$$
\frac{\partial(\rho u)}{\partial x}+\frac{\partial(\rho v)}{\partial y}=0
$$

The Navier-Stokes equations:

X-momentum:

$$
\frac{\partial(\rho u u)}{\partial x}+\frac{\partial(\rho u v)}{\partial y}=-\frac{\partial P}{\partial x}+\frac{\partial}{\partial x}\left(\mu \frac{\partial u}{\partial x}\right)+\frac{\partial}{\partial y}\left(\mu \frac{\partial u}{\partial y}\right)
$$

y-momentum:

$$
\frac{\partial(\rho u v)}{\partial x}+\frac{\partial(\rho v v)}{\partial y}=-\frac{\partial P}{\partial y}+\frac{\partial}{\partial x}\left(\mu \frac{\partial v}{\partial x}\right)+\frac{\partial}{\partial y}\left(\mu \frac{\partial v}{\partial y}\right)
$$

Energy:

$$
\frac{\partial}{\partial x}(\rho i u)+\frac{\partial}{\partial y}(\rho i v)=-p\left(\frac{\partial u}{\partial x}+\frac{\partial v}{\partial y}\right)+\frac{\partial}{\partial x}\left(k \frac{\partial T}{\partial x}\right)+\frac{\partial}{\partial y}\left(k \frac{\partial T}{\partial y}\right)+\Phi
$$


where $i$ is the specific internal energy and $\Phi$ is the short notation for the viscous-dissipation function. Since all terms are quadratic, viscous dissipation is always positive, so that a viscous flow always tends to lose its available energy due to dissipation. The governing equations for incompressible flow with constant thermophysical properties can be expressed as:

Continuity:

$$
\frac{\partial(\rho u)}{\partial x}+\frac{\partial(\rho v)}{\partial y}=0
$$

The Navier-Stokes equations:

x-momentum:

$$
\rho\left(u \frac{\partial u}{\partial x}+v \frac{\partial u}{\partial y}\right)=-\frac{\partial p}{\partial x}+\mu\left(\frac{\partial^{2} u}{\partial x^{2}}+\frac{\partial^{2} u}{\partial y^{2}}\right)
$$

y-momentum:

$$
\rho\left(u \frac{\partial v}{\partial x}+v \frac{\partial v}{\partial y}\right)=-\frac{\partial p}{\partial y}+\mu\left(\frac{\partial^{2} v}{\partial x^{2}}+\frac{\partial^{2} v}{\partial y^{2}}\right)
$$

Energy:

$$
\rho c_{p} u \frac{\partial T}{\partial x}=\kappa\left(\frac{\partial^{2} T}{\partial x^{2}}+\frac{\partial^{2} T}{\partial y^{2}}\right)+\mu\left(\frac{\partial u}{\partial y}\right)^{2}
$$

For fully developed steady incompressible flow between two parallel plates with constant thermophysical properties

$$
\frac{\partial u}{\partial x}=0 \text { or } u=u(y) \text { and } v=0
$$

The continuity equation is automatically satisfied and the Navier-Stokes can be written in Cartesian form as

$$
\begin{aligned}
& -\frac{1}{\rho} \frac{\partial p}{\partial x}+\frac{\mu}{\rho} \frac{\partial^{2} u}{\partial y^{2}}=0 \\
& -\frac{1}{\rho} \frac{\partial p}{\partial y}=0
\end{aligned}
$$

From Eqn. (8), it is can be concluded that the pressure $p=p(x)$.

The Equ. (7), can be rewritten as

$$
\frac{\partial^{2} u}{\partial y^{2}}=\frac{1}{\mu} \frac{\partial p}{\partial x}
$$

The energy equation reduces to 


$$
\rho c_{p} u \frac{\partial T}{\partial x}=\kappa\left(\frac{\partial^{2} T}{\partial x^{2}}+\frac{\partial^{2} T}{\partial y^{2}}\right)+\mu\left(\frac{\partial u}{\partial y}\right)^{2}
$$

In case of incompressible flow there is no need to establish linkage between the energy equation and the equations of continuity and momentum.

\subsection{Boundary Conditions}

In our simulation we considered the following boundary conditions for the parallel plate channel and are specified as follows:

(a) Inlet boundary: The velocity and temperature distribution at the inlet boundary is assumed to be uniform and parallel to the $\mathrm{x}$-axis, i.e.

$$
\frac{\partial u}{\partial x}=0, v=0 \text { and } T=T_{i} \text { at } x=0 .
$$

(b) Wall boundary: For noslip condition with stationary wall, $u=0$ and $v=0$ for both the walls. For slip condition

$$
u=u_{s}, v=0 \text { and } T=T_{s} \text { at } y=H / 2 \text { or } y=-H / 2 \text {, }
$$

where $u_{s}$ is the velocity slip and $T_{s}$ is the temperature jump of the fluid.

Since the flow is symmetric about the centerline of the channel

$$
\frac{\partial u}{\partial y}=0, v=0 \text { and } \frac{\partial T}{\partial y}=0 \text { at } \mathrm{y}=0
$$

The slip velocity condition was proposed by Maxwell [6] as

$$
u_{g}-u_{w}=\frac{2-\sigma_{m}}{\sigma_{m}} K n \frac{\partial u}{\partial y}
$$

and the temperature jump boundary conditions by Smoluchowski [7] as

$$
T_{g}-T_{w}=2\left(\frac{2-\sigma_{T}}{\sigma_{T}}\right) K n \frac{\partial T}{\partial y}
$$

where $\frac{\partial u}{\partial y}$ and $\frac{\partial T}{\partial y}$ are the variation of velocity and temperature normal to the wall. We considered the tangential-momentum-accommodation coefficient $\sigma_{m}=1$ and the thermal-accommodation coefficient $\sigma_{T}=1$ which describe the gas-wall interactions.

According to HS model gas molecules, continuously strike and reflect from a solid surface, just as they continuously collide with each other. For a perfectly smooth wall, the incident angle exactly equals the reflected angle. This is called specular reflection $\left(\sigma_{m}=0\right)$, where the tangential momentum of the molecules reflected from the wall is unchanged, but the normal momentum of 
the molecules is reversed due to the normal momentum transfer to the wall. In this case there is no tangential momentum exchange of the molecules with the wall and the molecules exert no shear on the wall. On the other hand, for an extremely rough wall the molecules reflect at some random angle uncorrelated to their incident angle. This is termed as diffuse reflection $\left(\sigma_{m}=1\right)$ where the molecules reflect from the wall with zero tangential momentum and the momentum is transmitted to the wall. Slip velocity is for the momentum balance at the wall. As above $\sigma_{T}=0$ corresponds to the no energy exchange and $\sigma_{T}=1$ corresponds to perfect energy exchange of the molecules with the wall.

The tangential-momentum-accommodation coefficient is defined by $\sigma_{m}=\frac{\tau_{i}-\tau_{r}}{\tau_{i}-\tau_{w}}$, where $\tau_{i}$ and $\tau_{r}$ indicate the tangential momentum of incoming and reflected molecules respectively and $\tau_{w}$ is the tangential momentum of re-emitted molecules.

The thermal-accommodation coefficient is defined by $\sigma_{T}=\frac{d E_{i}-d E_{r}}{d E_{i}-d E_{w}}$, where $d E_{i}$ and $d E_{r}$ indicate the energy fluxes of incoming and reflected molecules respectively and $d E_{w}$ is the energy flux if all the incoming molecules had been reemitted with the energy flux corresponding to the wall temperature.

Wall heating condition with constant wall temperature was implied on both the walls. The free stream temperature was $320 \mathrm{~K}$ and the difference of free stream temperature and the wall temperature was $30 \mathrm{~K}$. Pressure condition was assumed on both the inlet and outlet boundaries.

\subsection{Numerical Method}

The pressure based segregated solver was employed in order to achieve steady state analysis. In the present study, the governing equations are discretized using a control volume based technique. The governing equations are integrated about each control volume to get the discrete equations that conserve each quantity on a control-volume basis. Eqn. (13) shows the integration of the conservation equation of a scalar $\phi$ over an arbitrary control volume $V$.

$$
\int \rho \phi \vec{V} \cdot d \vec{A}=\int \Gamma_{\phi} \Delta \phi \cdot d \vec{A}+\int_{V} S_{\phi} d V
$$

where,

$\Gamma_{\phi}$ is the diffusion coefficient for $\phi$.

$\mathrm{S}_{\phi}$ is the rate of generation of $\phi$ per unit volume.

Equation (13) is applied to each control volume or cell in the computational domain. The Eqn. (13) is discretized on each cell of the domain and obtained Eqn. (14)

$$
\sum_{f}^{N \text { faces }} \rho_{f} \vec{V}_{f} \phi_{f} \cdot \vec{A}=\sum_{f}^{N \text { faces }} \Gamma_{\phi}(\nabla \phi)_{n} \cdot \vec{A}_{f}+S_{\phi} V
$$

where,

$N_{\text {faces }}$ is the number of faces enclosing a cell.

$\phi_{f}$ is the value of $\phi$ convected through $f$ 
By default, all calculated variable values $\phi$ are stored at the cell centers. But the face values $\phi_{f}$ are required to discretize the convection terms in Eqn. (14). The face values are interpolated from cell center values using second order upwind scheme. In this scheme face value $\phi_{f}$ is derived from quantities in the cell upstream of the face.

The problem associated with pressure velocity coupling is resolved by adopting an iterative solution strategy SIMPLE (Semi-Implicit Method for Pressure-Linked Equations) algorithm.

To initiate the SIMPLE calculation process a pressure field $p^{*}$ is guessed. Discretized $x$-momentum and y-momentum equations are solved using the guessed pressure field to yield velocity components $u^{*}$ and $v^{*}$. Then we defined the correction $p^{\prime}$ as the difference between correct pressure field $p$ and the guessed pressure field $p^{*}$, so that

$$
p=p^{*}+p^{\prime}
$$

Similarly we defined velocity corrections $u^{\prime}$ and $v^{\prime}$ to relate the correct velocities $u$ and $v$ to the guessed velocities $u^{*}$ and $v^{*}$ :

$$
\begin{aligned}
& u=u^{*}+u^{\prime} \\
& v=v^{*}+v^{\prime}
\end{aligned}
$$

Substitution of the correct pressure field $p$ into the momentum equations yields the correct velocity field $(u, v)$. Discretized $x$-momentum and $y$-momentum equations link the correct velocity fields with the correct pressure field.

The computations were considered to be converged when the residues for continuity, momentum and energy are less than $10^{-6}$.

\subsection{Grid Independency Test}

To evaluate the grid size effect, grid independency tests were carried out. Three different sizes of grid $51 \times 1201,61 \times 1501$ and $71 \times 1801$ were tested for a typical channel of $A R=300$ with slip boundary conditions. The relative difference of average velocity at cross section $x / L=0.9$ for grid size $51 \times 1201$ and $61 \times 1501$ is $0.06 \%$ and for grid size $61 \times 1501$ and $71 \times 1801$ is $0.05 \%$ respectively. The corresponding friction constant difference is $0.04 \%$ and $0.02 \%$. For convenience, we used mesh number $61 \times 1501$ or its multiple according to the length of the dimension of the domain.

\subsection{Results and Discussion}

To validate our simulation with the experimental results introduced by Pong et al. [9], we first simulated compressible slip, compressible noslip and incompressible noslip Nitrogen flow in a long parallel plate channel with channel height $1.2 \mu \mathrm{m}$ and length $3000 \mu \mathrm{m}$. The outlet was atmospheric pressure condition with pressure ratio (inlet/outlet) 2.36. The experimental investigation by Pong et al. [9] indicates that the pressure distribution in the slip flow regime is nonlinear. 


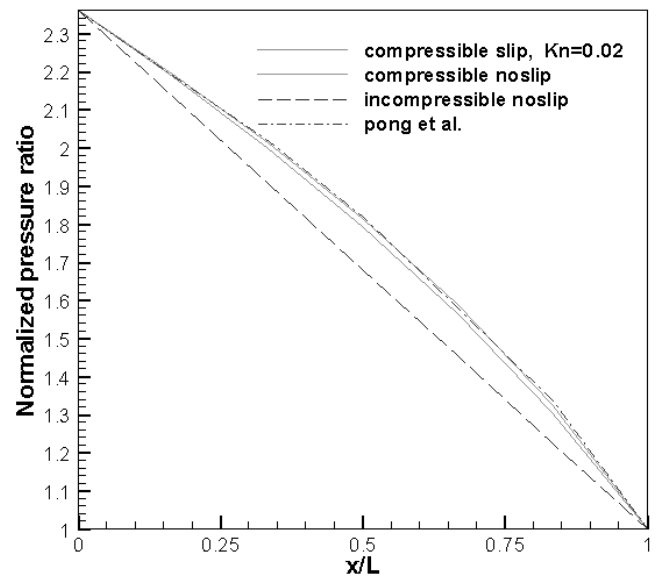

Fig. 2. Normalized pressure distribution along the centerline of the channel.

For further validation the friction constant $(f R e)$ for incompressible noslip flow is evaluated. In our simulation it is 96 which comply with that of Kakac et al. [13].

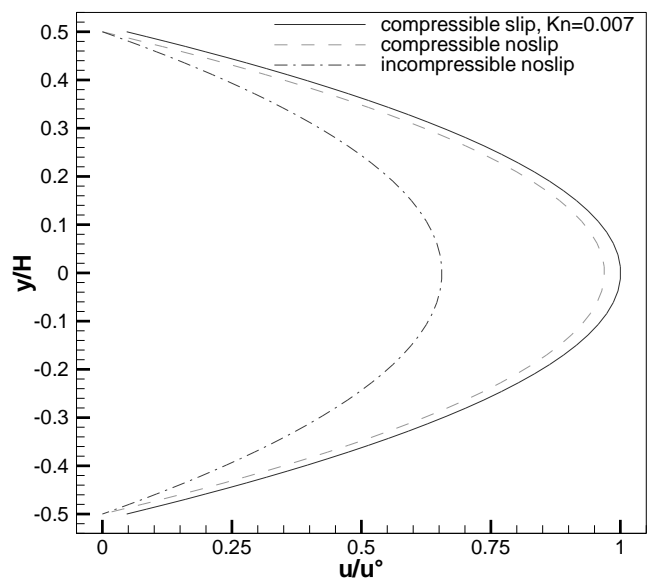

Fig. 3. Normalized velocity distribution on cross section at $x / L=0.9$.

We plotted the centerline longitudinal normalized pressure distribution for compressible slip, compressible noslip and incompressible noslip flows along with the results obtained by Pong et al. [9] in Fig. 2. The figure shows that our results agree well with that of Pong et al. [9]. The results indicate that the pressure distribution is nonlinear for both compressible slip and compressible noslip flows. For compressible flow, the density decreases along the downstream direction, as a result velocity increases and the pressure distribution is nonlinear.

The curvature of pressure distribution of compressible noslip is higher than that of compressible slip flow. The pressure distribution of incompressible noslip flow is linear. This figure shows that rarefaction (due to $K n$ ) and compressibility (due to $M a$ ) have opposite effects on the pressure distribution. Rarefaction effects reduce the curvature in pressure distribution which is the results of compressibility effects. 
The normalized velocity distribution of compressible slip, compressible noslip and incompressible noslip flows on cross section at $x / L=0.9$ along streamwise direction at pressure ratio 3.0 are depicted in Fig. 3. The compressible slip flow shows the highest velocity due to slip effect and the incompressible noslip flow shows the lowest velocity.

Table 1. Average velocity at $x / L=0.9$ different pressure ratios

\begin{tabular}{|c|c|c|c|c|}
\hline \multicolumn{2}{|c|}{} & \multicolumn{2}{c|}{ Average velocity } & \multirow{2}{*}{ Velocity difference } \\
\cline { 3 - 5 } \multicolumn{2}{|c|}{} & compressible slip & compressible noslip & \multirow{2}{*}{0.758075} \\
\hline \multirow{3}{*}{ Pressure ratio } & 2.0 & 11.866894 & 11.108819 & 1.407776 \\
\cline { 2 - 5 } & 3.0 & 26.419555 & 25.011779 & 1.918732 \\
\cline { 2 - 5 } & 4.0 & 41.211771 & 39.293039 & \\
\hline
\end{tabular}

Table 1 and Table 2 Show that on cross section at $x / L=0.9$, the differences of average velocity between compressible slip, compressible noslip for pressure ratios 2, 3 and 4 are 0.758075 , 1.407776 and 1.918732 respectively which are equivalent to the rate of increase of velocity by $6.82 \%, 5.63 \%$ and $4.88 \%$ respectively. Hence it is clear that the rate of increase of velocity in case of compressible noslip flow is higher than that of compressible slip flow.

Table 2. Average velocity at $x / L=0.9$ for different pressure ratios

\begin{tabular}{|c|c|c|c|c|}
\hline \multicolumn{2}{|c|}{} & \multicolumn{2}{c|}{ Average velocity } & \multirow{2}{*}{ Velocity difference } \\
\cline { 3 - 4 } \multicolumn{2}{|c|}{} & compressible noslip & incompressible noslip & \multirow{2}{*}{} \\
\hline \multirow{3}{*}{ Pressure ratio } & 2.0 & 11.108819 & 8.4576962 & 2.651123 \\
\cline { 2 - 4 } & 3.0 & 25.011779 & 16.906318 & 8.105461 \\
\cline { 2 - 4 } & 4.0 & 39.293039 & 25.345835 & 13.9472 \\
\hline
\end{tabular}

On the other hand these differences for compressible noslip and incompressible noslip are $2.651123,8.105461$ and 13.9472 respectively which is equivalent to the rate of increase of velocity by $31.35 \%, 47.94 \%$ and $55.03 \%$ respectively. This shows that as the pressure ratio increases, the rate of increase of average velocity difference between compressible noslip and incompressible noslip flow decreases whereas the rate of increase of average velocity difference between compressible slip and compressible noslip flow increases.

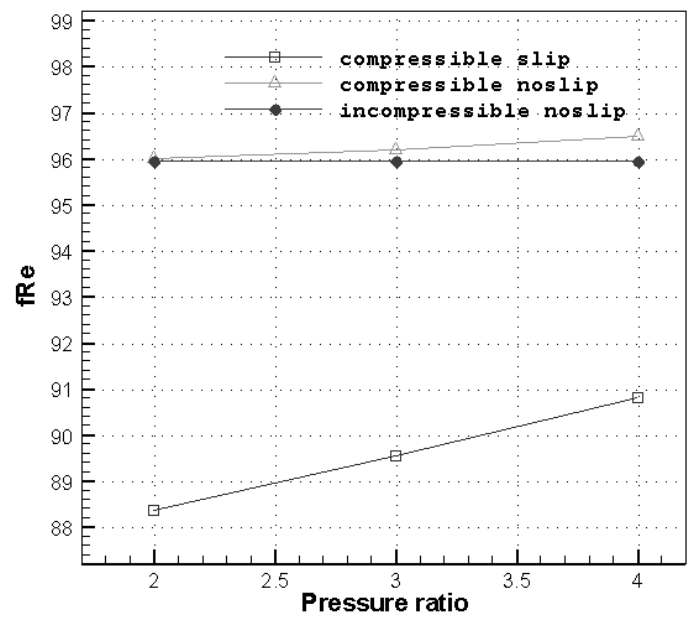

Fig. 4. The variation of friction constants at $x / L=0.9$ for different pressure ratios. 
We investigated the friction constant ( $f \mathrm{Re}$ ) for compressible flows with slip and noslip boundary conditions and for incompressible flow with noslip boundary condition for pressure ratios 2, 3 and 4. The results are depicted in Fig. 4.

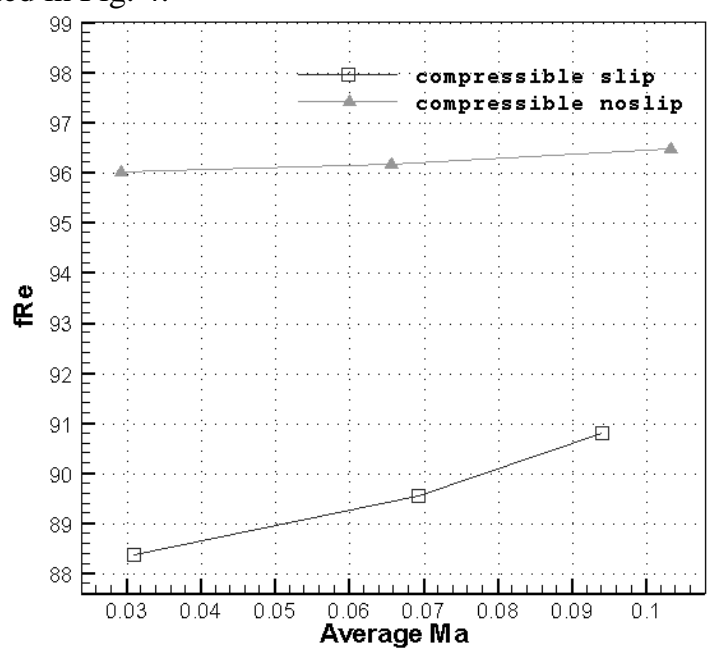

Fig. 5. The effect of average Mach number on friction constant at $x / L=0.9$

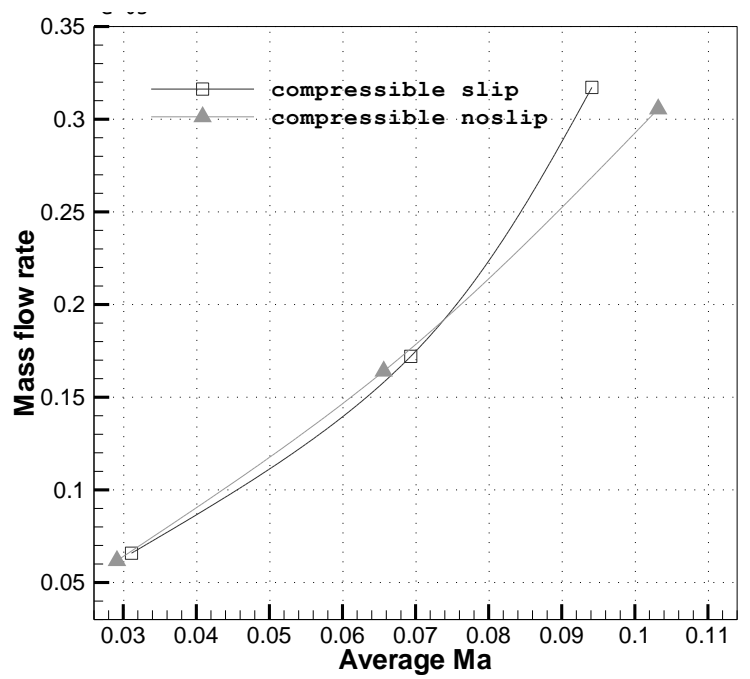

Fig. 6. The effect of average Mach number at $x / L=0.9$ on mass flow rate.

The results show that the friction constants are the highest for noslip compressible flow and lowest for the slip flow in parallel plate channels but the rate of increase of friction constant for slip flow is the highest. The friction constant increases about $121.68 \%$ for compressible slip flow and $16.04 \%$ for compressible noslip flow for the variation of pressure ratios from 2 to 4 .

For noslip incompressible flow the friction constant does not change for pressure variation and it is fixed to 96. This shows that the pressure ratio has strong influence on $f$ Re in compressible slip flow. 
Fig. 5 shows the variations of friction constants with respect to the variation of Mach numbers from 0.031 to 0.094 which results for the variation of pressure ratios from 2 to 4 . The friction constants for slip flow are lower than that of noslip compressible flow. The rate of increase of friction constant for slip flow is $2.75 \%$ with respect to Mach number, ranges from 0.031 to 0.094 . The corresponding increase for the case of noslip flow is about $0.0015 \%$ which is negligible.

Fig. 6 shows the effect of Mach number on mass flow rate. The mass flow rate of compressible noslip flow is a little bit higher than compressible slip flow for the variation of Mach number from 0.03 to 0.1 . The mass flow rate for compressible slip flow increases rapidly with the increase of Mach number and for Ma $>0.07$, the mass flow rate of compressible slip flow is higher than that of compressible noslip flow. The results depicted in Figs. 7 - 10 are obtained considering constant pressure ratio 2 and constant channel height $20 \mu \mathrm{m}$. We only changed the channel length from $750 \mu \mathrm{m}$ to $6000 \mu \mathrm{m}$.

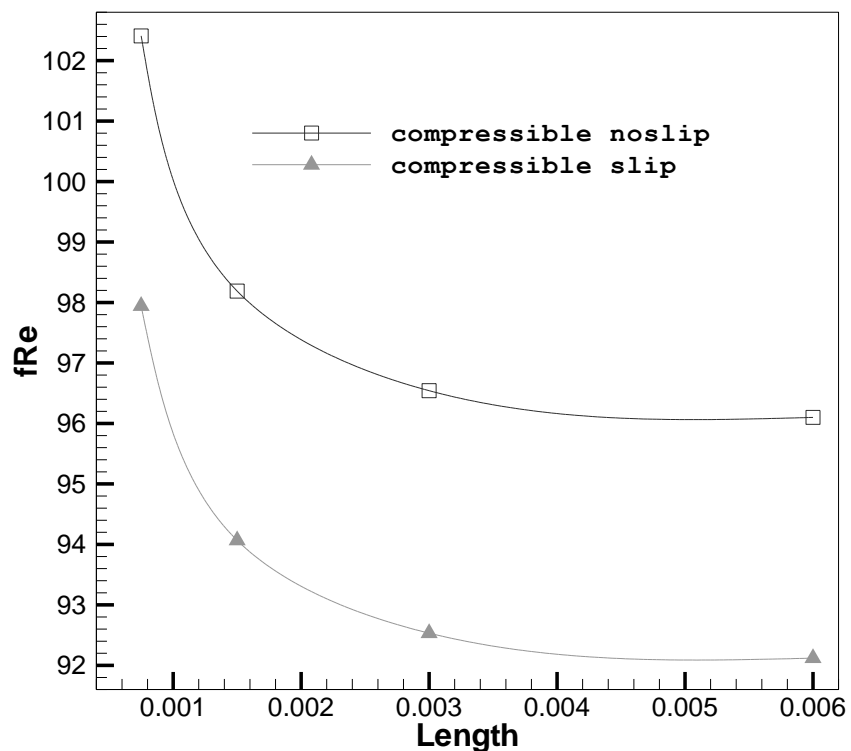

Fig. 7. The effect of channel length on friction constant at $x / L=0.9$.

The effect of channel length on frication constant $f R e$ is depicted by Fig. 7. The figure shows that as the channel length increases, the friction constant decreases for both compressible slip and noslip flows. The decrease in case of compressible slip is rapid in comparison to compressible noslip flow. It is evident from the figure that as the channel length increases, $f R e$ decreases continuously for compressible slip flow but it approaches to an asymptotic value of 96 for compressible noslip flow.

Fig. 8 shows the influence of Mach number on normalized friction constant. The friction constant was normalized by the friction constant of incompressible noslip flow. If the normalized friction constant (compressible-fRe/incompressible-fRe) is greater the 1 , we can consider that flow as compressible. 


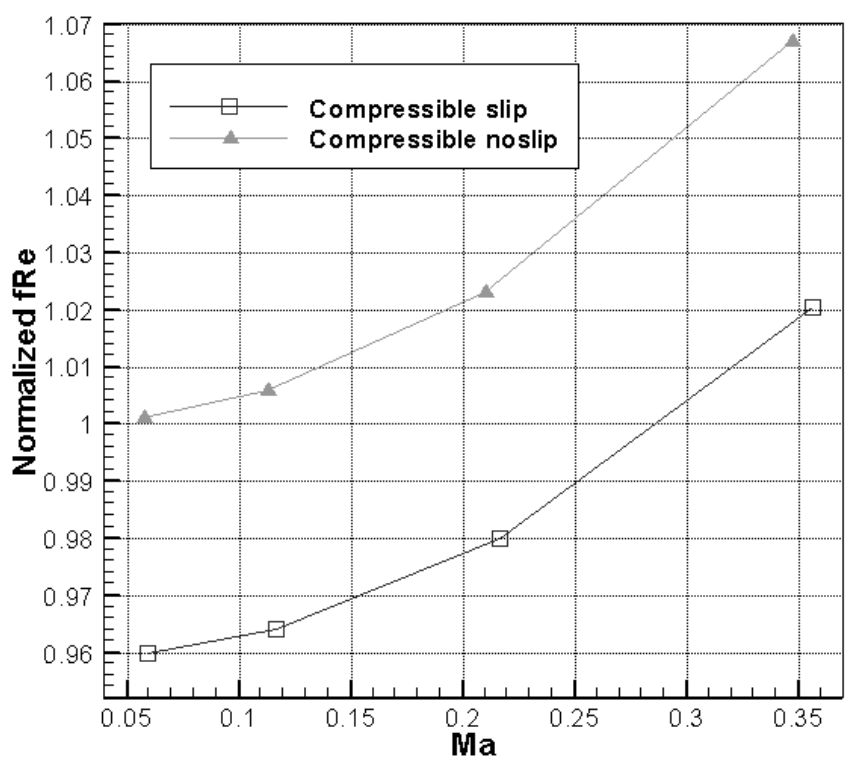

Fig. 8. The effect of average Mach number on friction constant at $x / L=0.9$.

From figure we see that the compressible noslip flow shows compressibility effect when the Mach number is 0.05 which is very small in compare to its conventional counterpart 0.3 and this may be due to the effect of small dimensions of the microchannel. The figure also shows that compressible slip flow shows compressibility effect when Mach number is 0.28 . If we look at the result displayed in Fig. 4 and Fig. 5, we see that $f R e$ can be described better by pressure ratio than by Mach number.

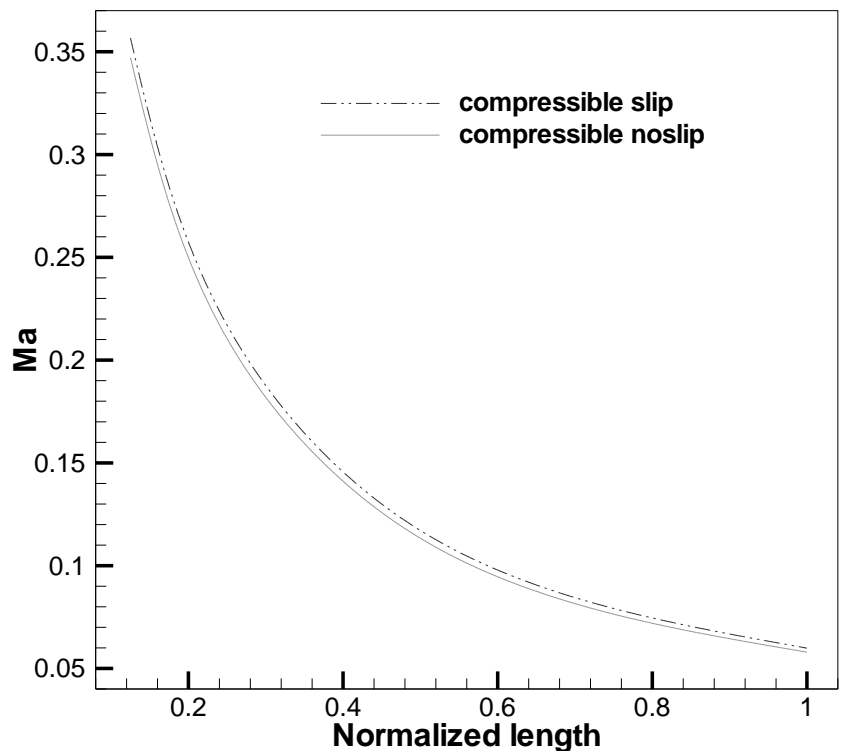

Fig. 9. The effect channel length on average Mach number at $x / L=0.9$. 


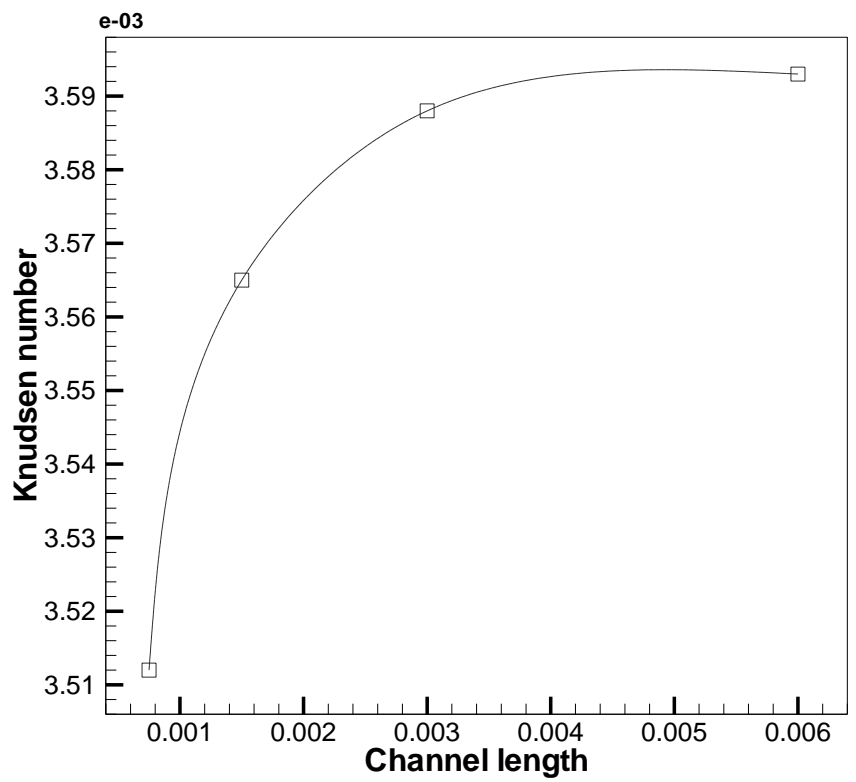

Fig. 10. The effect channel length on Knudsen number at $x / L=0.9$.

Normalized channel length and Mach numbers are displayed in Fig. 9. As the $A R$ increases due to increase of channel length, the Mach number decreases rapidly for both compressible slip and compressible noslip flows. For the same $A R$, the Mach number for compressible slip flow is higher than that of compressible noslip flow.

Fig. 10 shows the variation of Knudsen number with change of channel length. The Knudsen number increases with the increase of channel length but the increase of $K n$ is very small. If the length is increased 8 times then the $K n$ increases only by $2.33 \%$.

\section{Conclusions}

Two dimensional compressible and incompressible momentum and energy equations were solved with slip and noslip boundary conditions for the parallel plate microchannels. The results can be concluded as follows:

The pressure distribution for slip and noslip compressible flows are nonlinear where as it is linear for noslip incompressible flow in micarochannels. This nonlinearity is due to compressibility. The nonlinearity of compressible slip flow is a little bit lower than that of noslip compressible flow because of lower friction on the wall. The friction constant for compressible slip flow is lower than that of compressible noslip flow for the effect of both pressure ratio and Mach number. But the rate of increase of friction constant for slip flow is higher than that of compressible noslip flow. As the $A R$ increases, the friction constant decreases continuously for compressible slip flow but it approaches to an asymptotic value of 96 for compressible noslip flow. In parallel micro channel flow, fRe can be described better by pressure ratio than by Mach number. 


\section{REFERENCES}

[1] Giacomo Puccetti, Beatrice Pulvirenti, Gian Luca Morini, "Experimental determination of the 2D velocity laminar profile in glass microchannels using $\mu$ PIV", Energy Procedia, 45(2014), pp.538-547.

[2] Ines Hamidi and Abdelmottaleb Ouederni, "Single phase flow characteristics in rectangular microchannel: entrance length and friction factor", International Journal of Innovation and Applied Studies, 8 (2014), pp. 819-826.

[3] Khaleel Khasawneh And Chunpei Cai, "High Order Slip Boundary Solutions For Gaseous Flow In A Uniform Rectangular Microchannel”, Journal of Fluids and Thermal Sciences, 1 (2012), pp.71-83.

[4] V. M. Aniskin, K. V. Adamenko, A. A. Maslov, "Experimental determination of the friction factor coefficient in microchannels", Journal of Applied Mechanics and Technical Physics, 52 (2011), pp. $18-23$.

[5] George Em Karniadakis and Ali Beskok, "Micro Flows Fundamentals and Simulation", SpringerVerlag New York Inc, 2002.

[6] Maxwell, J.C., "On Stresses in Rarified Gases Arising From Inequalities of Temperature”, Philos. Trans. R. Soc. London, 170 (1879), pp. 231-256.

[7] Smoluchowski, M., "Über den Temperatursprung bei Wärmeleitung in Gasen", Akad. Wiss. Wien. CVII (1898), pp. 304-329.

[8] Errol R. Arkilic, Martin A. Schmidt, and Kenneth S. Breuer, "Gaseous Slip Flow in Long Microchannels”, Journal of Microelectromechanical Systems, 6 (1997), pp. 167-178.

[9] Pong, K. C., Ho, C. M. Liu, J. and Tai, Y. C., "Non-linear Pressure Distribution in Uniform Microchannels", Application of Microfabrication to Fluid Mechanics, ASME, 197 (1994), pp. 51-56.

[10] Ali Beskok and George E. Karniadakis, "Simulation of Heat and Momentum Transfer in Complex Microgeometries", Journal of Thermophysics and Heat Transfer, 8 (1994), pp. 647-655.

[11] Schaaf, S. and Chambre, P., "Flow of Rarefied Gases", Princeton University Press, Princeton, 1961.

[12] Mohamed Gad-el-Hak, "The MEMS Handbook (2 $2^{\text {nd }}$ Edition)", Taylor and Francis Group, New York, 2006.

[13] R. Prud'homme, T. Chapman, and J. Bowen, "Laminar Compressible Flow in a Tube", Appl. Sci. Res., 43 (1986), pp. 67-74.

[14] Kakac, S, Shah, R. K. and Aung, W., "Handbook of Single-Phase Convective Heat Transfer", John Wiley \& Sons, New York, 1987.

[15] H. van den Berg, C. Seldam, and P.Gulik, "Compressible Laminar flow in a capillary", J. Fluid mech., 246 (1993), pp. 1-20. 